\title{
Impaired adaptation to left atrial pressure increase in patients with atrial fibrillation
}

\author{
Gergely Ágoston $^{1}$ - Judit Szilágyi ${ }^{1}$ - Gábor Bencsik ${ }^{1}$ - Cristina Tutuianu ${ }^{1}$. \\ Gergely Klausz $^{1} \cdot$ László Sághy $^{1} \cdot$ Albert Varga $^{1} \cdot$ Tamás Forster $^{1} \cdot$ Róbert Pap $^{1}$
}

Received: 11 April 2015 / Accepted: 15 June 2015 /Published online: 30 June 2015

(C) Springer Science+Business Media New York 2015

\begin{abstract}
Background or purpose Episodes of left atrial (LA) pressure increase predispose to atrial fibrillation (AF). The adaptation of LA mechanical function and electrophysiology to pressure elevation in healthy adults, and in patients with AF, is largely unknown.

Methods Eleven patients with left-sided accessory pathway (controls) and 16 patients with paroxysmal AF undergoing catheter ablation were studied. LA pressure (LAP) was recorded through transseptal catheterization, while speckle trackingderived peak LA longitudinal strain (PALS) was measured using transthoracic echocardiography. Stiffness index (SI) was calculated as mean LAP/PALS. Effective refractory period (ERP) of the LA was determined during simultaneous atrioventricular (AV) pacing and during atrial pacing.

Results At baseline, AF patients had higher LA pressure (mean LAP $8.3 \pm 4.7$ vs. $5.1 \pm 3.1 \mathrm{mmHg}, p=0.048$ ), reduced LA mechanical function (PALS $15.1 \pm 5.1$ vs. $21.6 \pm 6.2 \%$, $p=0.006$, SI $0.69 \pm 0.75$ vs. $0.28 \pm 0.22, p=0.015$ ), and longer LA ERP $(242.3 \pm 33.4$ vs. $211.7 \pm 15.6 \mathrm{~ms}, p=0.017)$. Mean LAP was increased to the same extent by AV pacing in controls and AF patients (mean change $12.6 \pm 7.4$ vs. $12.6 \pm$ $7.5 \mathrm{mmHg}, p=0.980$ ). At the same time PALS decreased (from $15.1 \pm 5.1$ to $11.6 \pm 3.3 \%, p=0.008$ ), SI increased (from $0.69 \pm 0.75$ to $1.29 \pm 1.17, p<0.001$ ) and ERP shortened (from $242.3 \pm 33.4$ to $215.9 \pm 26.3 \mathrm{~ms}, p=0.003$ ) in AF patients, while they remained unchanged in controls.
\end{abstract}

Róbert Pap

pap.paprobert@gmail.com

1 2nd Department of Medicine and Cardiology Centre, University of Szeged, Semmelweis str. 6., 6725 Szeged, Hungary
Conclusions The stiffened LA in patients with AF responds to acute pressure elevation with an exaggerated increase in wall tension and decrease in ERP, which is not seen in the normal LA. This may underlie the propensity for AF during episodes of atrial stretch in these patients.

Keywords Atrial fibrillation · Left atrial pressure · Mechanoelectric feedback $\cdot$ Left atrial strain

\section{Introduction}

Atrial pressure elevation predisposes to atrial fibrillation (AF) by several mechanisms. Acute atrial pressure increase leads to electrophysiologic changes, while chronic atrial stretch also induces structural remodeling.

In animal models, acute atrial dilatation has been shown to decrease atrial effective refractory period (ERP), result in slowing and block of impulse conduction and increased AF vulnerability [1-3]. This response has been termed atrial mechanoelectric feedback [4]. Similar changes have been described during acute pressure elevation by some [5-7] but not by others $[8-10]$ in the human right atrium. However, the relation between atrial pressure and ERP has not been studied in the human left atrium (LA), the major source of AF.

Structural changes occur in the atria of patients with septal defect, mitral valve disease, hypertension, and heart failure even before the first detected episode of AF [11-14]. These changes are mediated by increased hemodynamic load on the atria. Chronic atrial stretch-induced remodeling includes atrial dilatation, fibrosis, loss of contractile elements, and a propensity for AF [15]. Structural remodeling results in a decline of the reservoir function of the LA, which can be estimated by the echocardiographic measurement of peak atrial longitudinal strain (PALS) during ventricular systole. In patients with 
AF, PALS has been shown to correlate with the degree of LA fibrosis [16], thromboembolic risk [17], and the likelihood of sinus rhythm maintenance both after cardioversion [18] and catheter ablation [19]. However, the effect of LA pressure on PALS has not been determined.

Our aim was to study the responses in electrical (mechanoelectric feedback) and reservoir function to acute pressure elevation in the normal human LA and in the LA of patients with AF.

\section{Methods}

\subsection{Study group}

Consecutive patients with manifest or concealed left-sided accessory pathway, without a history of AF (controls) and patients with paroxysmal AF scheduled for pulmonary vein isolation, who had no symptomatic and/or documented $\mathrm{AF}$ episodes in the week prior to the procedure, were included. Exclusion criteria were persistent $\mathrm{AF}, \mathrm{CHADS}_{2}$ (Congestive Heart Failure, Hypertension, Age, Diabetes, Stroke [Doubled]) score $>2$, previous LA ablation or open heart surgery, heart failure, reduced left ventricular function, and moderate to severe mitral regurgitation.

\subsection{Electrophysiologic procedures}

Informed consent was obtained and antiarrhythmic drugs have been discontinued for at least five half-lives at the time of the procedure. Using right \pm left femoral vein access single (control patients) or double (AF patients) transseptal puncture was performed using 8.0 or 8.5 French transseptal sheaths (Fast-Cath, St. Jude Medical, St. Paul, MN, USA), under intracardiac echocardiographic guidance. The side arm of the transseptal sheath was connected to a disposable pressure transducer (Combitrans, B. Braun, Melsungen, Germany), which was positioned and zeroed at a reference level $5 \mathrm{~cm}$ below the left sternal border, at the fourth intercostal space [20]. Pressure was recorded at a sampling rate of $977 / \mathrm{s}$ by the CardioLab EP Recording System (GE Healthcare, Chalfont St Giles, UK).

\subsection{Pacing protocol}

The protocol was performed after the completion of the catheter ablation procedure, during the waiting period. At each site, pacing was performed with 2-ms stimulus duration, at twice diastolic threshold. Simultaneous atrioventricular (AV) pacing was carried out to produce an acute increase in LA pressure. LA ERP was determined both during simultaneous AV pacing and during atrial pacing at the same cycle length to control for the effect of the preceding cycle length on atrial ERP (Fig. 1). In AF patients, the atrial pacing catheter was positioned in the LA appendage; while in control patients, LA ERP was determined by pacing from the distal bipole of the coronary sinus (CS) catheter to avoid the need for a second transseptal puncture. This has been previously shown to reflect LA ERP well [21, 22]. Simultaneous AV pacing at a cycle length of $500 \mathrm{~ms}$ was carried out for at least $3 \mathrm{~min}$ to allow stabilization of pressure. Then, after every 30th drive stimulus progressively more premature (5-ms steps) atrial stimuli were introduced, without a pause in the drive train. LA ERP was defined as the longest coupling interval of the extrastimulus that failed to capture the atrium twice in succession.

\subsection{Echocardiographic measurements}

All patients underwent comprehensive two-dimensional transthoracic echocardiography examination using a commercially available ultrasound machine (Vivid I, GE Medical Systems, Horten, Norway) equipped with a $2.5-3.5-\mathrm{MHz}$ phased array transducer and software application for two-dimensional speckle tracking-based strain imaging.

LA volumes were calculated using the biplane method of disks (modified Simpson's rule), in the apical 4- and 2chamber view at end-systole (maximum LA size), and a mean value of volume was obtained [23]. LA volumes were indexed (LAVI) to body surface area (BSA). Mitral annular velocity was evaluated by tissue Doppler in the pulsed-wave mode [24].

\subsection{Assessment of left atrial reservoir function}

Particular attention was paid to obtain an adequate twodimensional-grayscale image, allowing obvious delineation of LA wall and extracardiac structures. The frame rate was set between 60 and 80 frames per second. Three consecutive heart cycles were recorded at baseline and immediately after simultaneous AV pacing (Fig. 2). Recordings were processed using acoustic-tracking software (EchoPac PC version 110.1.8, GE Healthcare, Horten, Norway), allowing off-line semiautomated analysis of speckle tracking-based strain [25]. In the end-diastolic/systolic frame, the atrial endocardial border was marked by a point-and-click method. After automatic creation of a region of interest, the LA wall was divided into six regions, and segmental tracking quality was analyzed (Fig. 2). The reference point was set at the onset of the QRS, and the average positive peak atrial longitudinal strain (PALS), which corresponds to LA reservoir function, was measured (Fig. 2). Values from the three consecutive cycles 
Fig. 1 Determination of LA effective refractory period (ERP) during atrial pacing (a) and during simultaneous $\mathrm{AV}$ pacing (b). $L A A$ LA appendage, $L A P$ left atrial pressure, $R V$ right ventricle. Coupling intervals (CI) of extrastimuli are shown in milliseconds. ERP is defined as the longest CI without atrial capture (a)

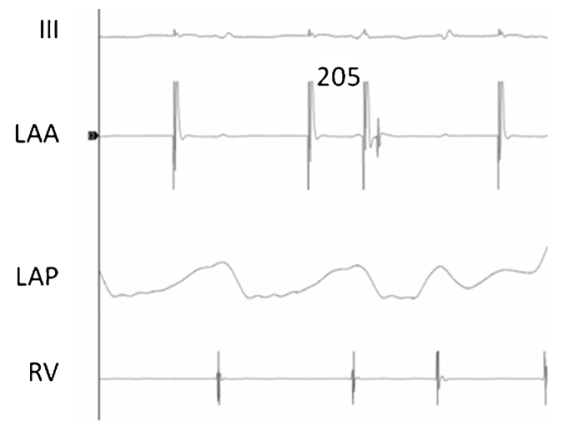

(b)

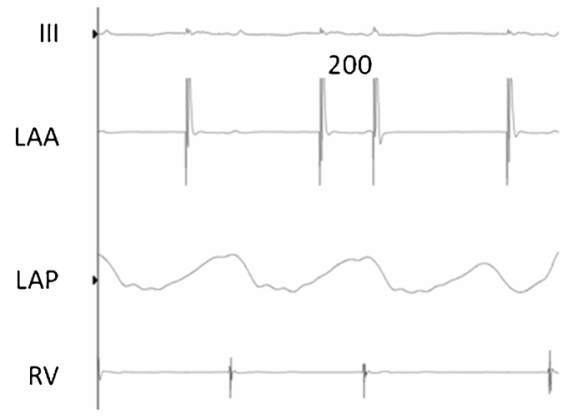

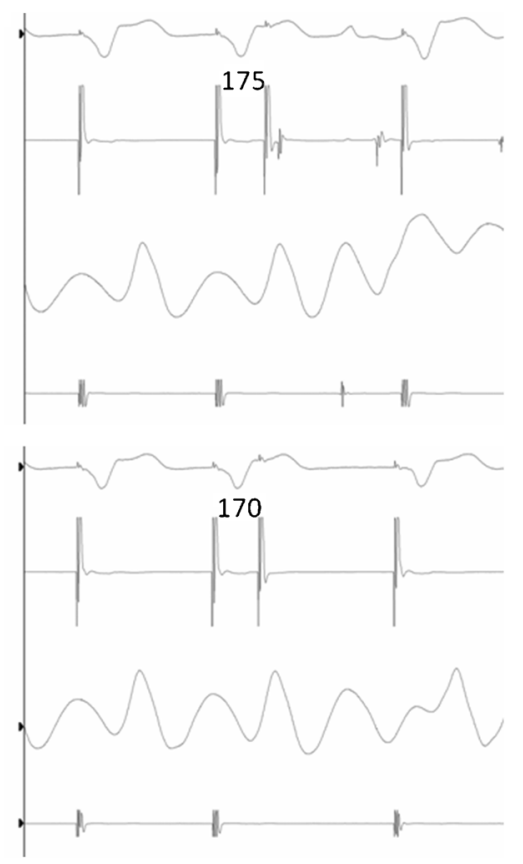

were averaged [26]. The LA stiffness index (SI) was calculated as mean LA pressure (LAP)/PALS [27].

\subsection{Statistical analysis}

Continuous variables are presented as mean \pm standard deviation and were tested for normality using the Kolmogorov-Smirnov test and compared by the Student's $t$ test or Mann-Whitney test as appropriate. Categorical variables are expressed as percentage and compared using the chi-square test. All statistical analyses were performed using SPSS software (SPSS Inc., Chicago, IL, USA). A $p$ value $<0.05$ was considered statistically significant.

\section{Results}

\subsection{Clinical characteristics and baseline values of the two groups}

Eleven patients undergoing left-sided accessory pathway ablation (controls) and 16 patients with paroxysmal AF were included. Controls were younger and had smaller LA volume index (LAVI), without further differences in clinical characteristics (Table 1).

Patients with AF had higher mean (mLAP) and peak (pLAP) invasive LA pressures at baseline ( $8.3 \pm 4.7$ vs. $5.1 \pm$ $3.1 \mathrm{mmHg}, p=0.048$ and $20.8 \pm 8.8$ vs. $14.6 \pm 5.7 \mathrm{mmHg}, p=$ 0.015 , respectively), compared to controls. Baseline LA PALS was significantly lower ( $15.1 \pm 5.1$ vs. $21.6 \pm 6.2 \%, p=0.006)$,

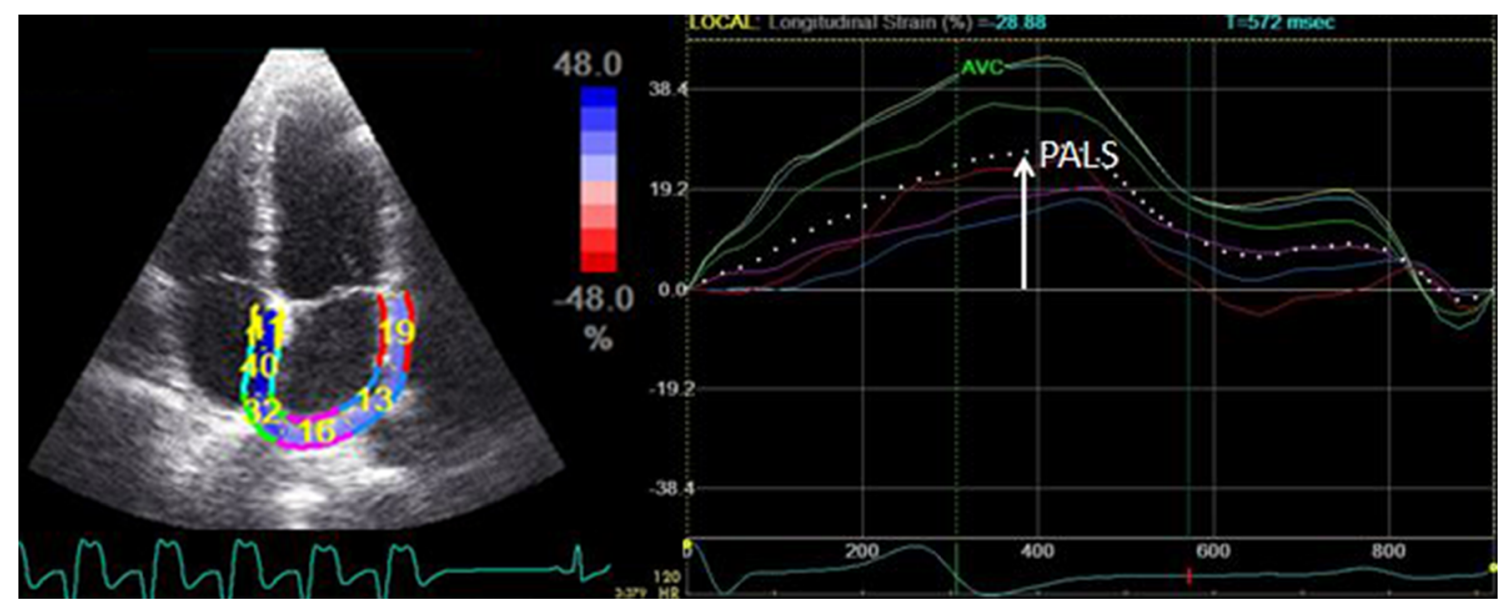

Fig. 2 Measurement of peak atrial longitudinal strain $(P A L S)$ immediately after simultaneous AV pacing. The dashed curve represents the average PALS 
Table 1 Baseline clinical and echocardiographic parameters

\begin{tabular}{|c|c|c|c|}
\hline & Controls & AF patients & $p$ value \\
\hline Age (year) & $42.2 \pm 21.1$ & $60.3 \pm 8.8$ & 0.019 \\
\hline Female (\%) & 22 & 31 & 0.629 \\
\hline $\operatorname{BSA}\left(\mathrm{m}^{2}\right)$ & $1.91 \pm 0.22$ & $1.98 \pm 0.22$ & 0.381 \\
\hline Hypertension (\%) & 27 & 56 & 0.137 \\
\hline Diabetes $(\%)$ & 0 & 0 & \\
\hline $\mathrm{CHADS}_{2}$ score & $0.22 \pm 0.44$ & $0.75 \pm 0.68$ & 0.084 \\
\hline LVEF (\%) & $64.7 \pm 6.8$ & $59.8 \pm 3.7$ & 0.189 \\
\hline $\mathrm{E}_{\mathrm{a}}(\mathrm{cm} / \mathrm{s})$ & $12.0 \pm 2.6$ & $10.4 \pm 3.3$ & 0.331 \\
\hline LAVI $\left(\mathrm{ml} / \mathrm{m}^{2}\right)$ & $32.4 \pm 11.4$ & $59.4 \pm 12.1$ & $<0.001$ \\
\hline
\end{tabular}

$B S A$ body surface area, $L V E F$ left ventricular ejection fraction, $E_{a}$ mitral annulus early diastolic velocity, LAVI LA volume indexed to BSA

while baseline SI was higher $(0.69 \pm 0.75$ vs. $0.28 \pm 0.22$, $p=0.015$ ), pointing to diminished LA reservoir function in patients with AF. At the same time, LA ERP was longer at baseline in AF patients, compared to controls (242.3 $\pm 33.4 \mathrm{vs.}$ $211.7 \pm 15.6 \mathrm{~ms}, p=0.017$ ).

\subsection{Left atrial pressure elevation}

During simultaneous AV pacing, mLAP rose by the same extent in controls and AF patients (mean change 12.6 \pm 7.4 vs. $12.6 \pm 7.5 \mathrm{mmHg}, p=0.980)$. At the same time, LA PALS
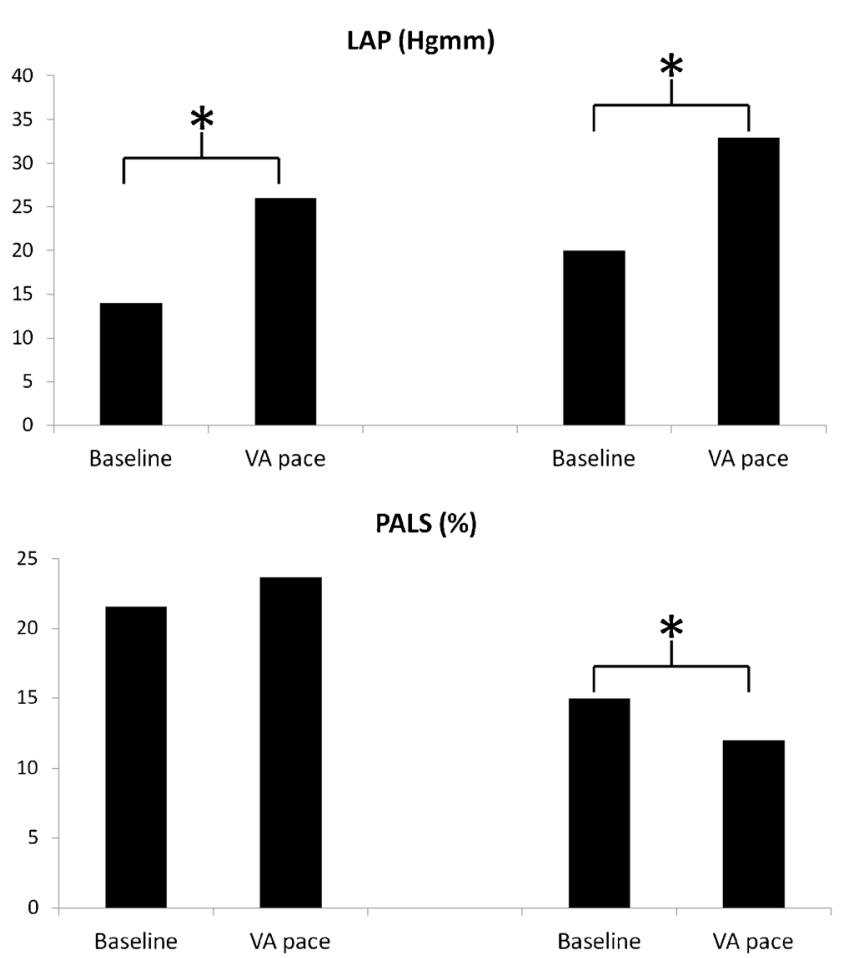

Control
PALS (\%)

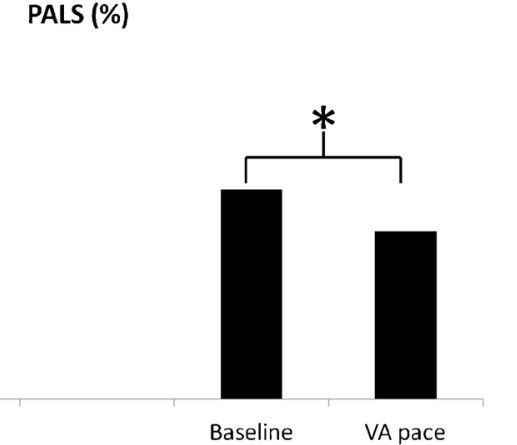

AF decreased (from $15.1 \pm 5.1$ to $11.6 \pm 3.3 \%, p=0.008$ ) and SI increased (from $0.69 \pm 0.75$ to $1.29 \pm 1.17, p<0.001$ ) in patients with AF, while they remained unchanged in controls (from $21.6 \pm 6.2$ to $22.9 \pm 7.1 \%, p=0.405$ and from $0.28 \pm 0.22$ to $0.45 \pm 0.43, p=0.10$, respectively). With pressure elevation, LA ERP decreased in AF patients (from 242.3 \pm 33.4 to 215.9 $\pm 26.3 \mathrm{~ms}, p=0.003$ ) but was not changed significantly in controls (from $211.9 \pm 16.7$ to $206.3 \pm 19.6 \mathrm{~ms}, p=0.276$ ) (Fig. 3).

\subsection{Follow-up}

Four of 16 AF patients (25\%) experienced arrhythmia recurrence after pulmonary vein isolation, during $16 \pm 7$ months of follow-up. Patients with recurrence had lower baseline LA reservoir function $(\mathrm{PALS}=10.7 \pm 3.2$ vs. $16.7 \pm 4.0 \%$, $p=0.036$ ), compared to those without.

\section{Discussion}

\subsection{Main findings}

We could not show the operation of mechanoelectric feedback or a change in the reservoir function of the normal LA during acute pressure elevation. We have seen, on the other hand, a dramatic fall in ERP and reservoir function in response to

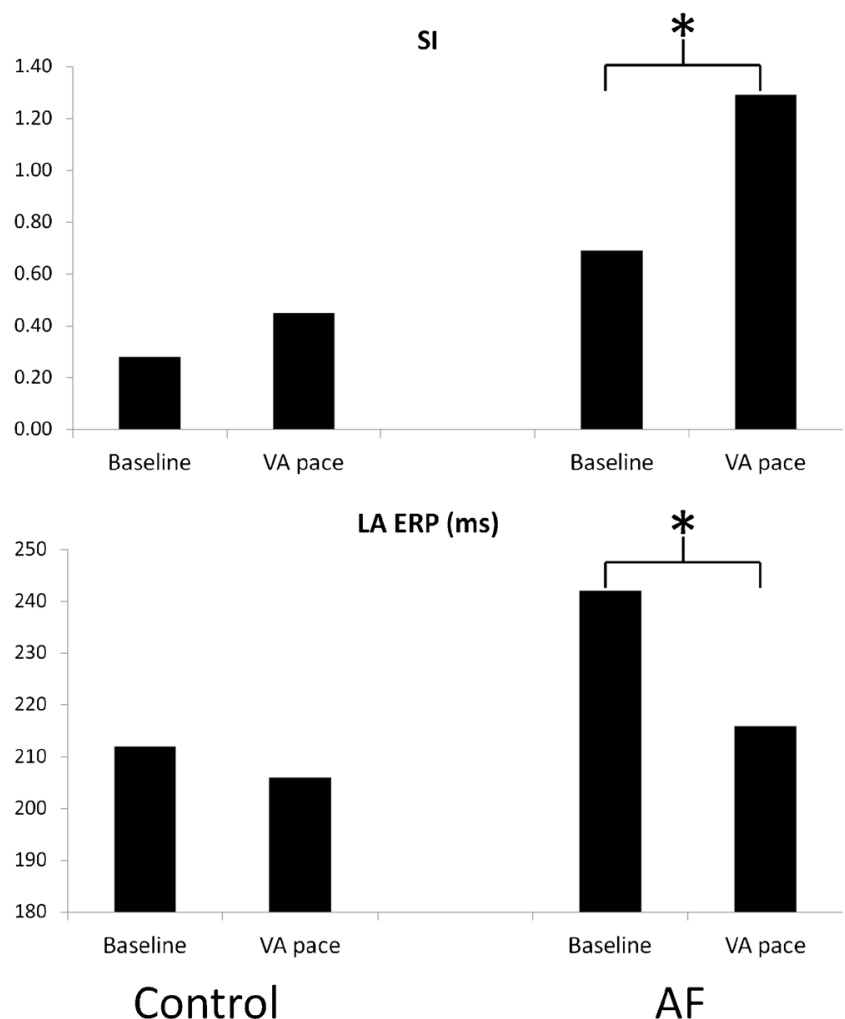

Fig. 3 Changes in mean LA pressure $(L A P)$, LA strain $(P A L S)$, stiffness index $(S I)$, and refractory period $(E R P)$ in response to simultaneous AV pacing in controls and AF patients. Stars mark significant $(p<0.05)$ changes 
pressure rise in patients with AF. We conclude that the normal adaptation to acute elevations in LA pressure is lost in patients with $\mathrm{AF}$, even during sustained sinus rhythm.

Even when in sinus rhythm, patients with paroxysmal AF show diminished LA reservoir function estimated by LA strain $[28,29]$. We have shown in this study that LA strain is also dependent on LA pressure in patients with $\mathrm{AF}$, and an acute rise in pressure leads to a decline in LA reservoir function and increased stiffness, a response not observed in the normal LA.

In patients with paroxysmal AF, but without a recent episode, LA ERP measured at the LA appendage has been shown by some [30,31], but not by other reports [32] to be longer than in controls, while it was consistently shorter in patients with persistent AF. The reason for this inconsistency might be the dependence of atrial refractoriness on pressure, a phenomenon known as mechanoelectric feedback [4].

Mechanoelectric feedback is well described in the ventricles, has been shown at the atrial level and in the human right atrium, but has not been studied in the human LA, the major source of AF [5-8, 33]. Acute atrial stretch increases vulnerability to AF in both animal models and humans $[1,34]$; the mechanism most commonly considered behind this is a shortening of refractoriness and slowing of impulse conduction $[2,4,6,35]$, both promoting the development of reentry. We have shown in this study that pressure-related shortening of refractoriness - mechanoelectric feedback-is magnified in the LA of patients with AF, which likely facilitates the persistence of the arrhythmia.

Paroxysmal AF itself leads to atrial pressure elevation [36]. According to our study, increased atrial pressure can result in increased stiffness and wall tension with shortened atrial refractoriness favoring AF maintenance. This way, a vicious circle is established, which may culminate in persistent AF.

\subsection{Limitations}

Due to inherent differences between the patient populations and procedures, the control and AF groups could not be well matched in all baseline characteristics. Therefore, the main outcome of this study was not the absolute value of electrical and mechanical parameters but rather the difference in the magnitude of pressure-related change between the two groups. Subjects in the control group were slightly younger, related to differences in the typical age of presentation of the two arrhythmias. Therefore, it cannot be excluded that some of the baseline differences between groups are also age-related. However, our paroxysmal AF population was otherwise relatively healthy and comparable in other clinical parameters to the control group, except for AF-related LA remodeling. The pacing protocol was carried out after ablation, during the waiting period for ethical reasons, to avoid prolonging left atrial access. It cannot be excluded that more extensive ablation in the AF group influenced the results. However, ablation in these patients was limited to the posterior LA, around the pulmonary veins (PVs), which is a region that is relatively immobile due to tethering by PVs, and this part of the LA is not included in echocardiographic strain analysis.

\section{Conclusions}

The normal LA can adapt to episodes of acute pressure elevation without a substantial change in reservoir function and ERP. On the other hand, patients with AF show an exaggerated fall in their already diminished LA reservoir function in response to pressure rise, with an out of proportion increase in wall tension leading to a decline in LA ERP, which likely further promotes the development of AF.

\section{References}

1. Solti, F., Vecsey, T., Kékesi, V., \& Juhász-Nagy, A. (1989). The effect of atrial dilatation on the genesis of atrial arrhythmias. Cardiovascular Research, 23(10), 882-886.

2. Ravelli, F., \& Allessie, M. (1997). Effects of atrial dilatation on refractory period and vulnerability to atrial fibrillation in the isolated Langendorff-perfused rabbit heart. Circulation, 96, 1686-1695.

3. Eijsbouts, S. C., Majidi, M., van Zandvoort, M., \& Allessie, M. A. (2003). Effects of acute atrial dilation on heterogeneity in conduction in the isolated rabbit heart. Journal of Cardiovascular Electrophysiology, 14, 269-278.

4. Nazir, S. A., \& Lab, M. J. (1996). Mechanoelectric feedback and atrial arrhythmias. Cardiovascular Research, 32(1), 52-61.

5. Calkins, H., el-Atassi, R., Kalbfleisch, S., Langberg, J., \& Morady, F. (1992). Effects of an acute increase in atrial pressure on atrial refractoriness in humans. Pacing and Clinical Electrophysiology, 15(11 Pt 1), 1674-1680.

6. Tse, H. F., Pelosi, F., Oral, H., Knight, B. P., Strickberger, S. A., \& Morady, F. (2001). Effects of simultaneous atrioventricular pacing on atrial refractoriness and atrial fibrillation inducibility: role of atrial mechanoelectrical feedback. Journal of Cardiovascular Electrophysiology, 12(1), 43-50.

7. Manios, E. G., Mavrakis, H. E., Kanoupakis, E. M., Kallergis, E. M., Kafarakis, P. K., \& Vardas, P. E. (2006). Evidence of mechanoelectric feedback in the atria of patients with atrioventricular nodal reentrant tachycardia. Journal of Interventional Cardiac Electrophysiology, 16(1), 51-57.

8. Klein, L. S., Miles, W. M., \& Zipes, D. P. (1990). Effect of atrioventricular interval during pacing or reciprocating tachycardia on atrial size, pressure, and refractory period. Contraction-excitation feedback in human atrium. Circulation, 82(1), 60-68.

9. Efremidis, M., Sideris, A., Prappa, E., Filippatos, G., Athanasias, D., Kardara, D., Sioras, I., \& Kardaras, F. (1999). Effect of atrial pressure increase on effective refractory period and vulnerability to atrial fibrillation in patients with lone atrial fibrillation. Journal of Interventional Cardiac Electrophysiology, 3(4), 307-310.

10. Chen, Y. J., Tai, C. T., Chiou, C. W., Wen, Z. C., Chan, P., Lee, S. H., et al. (1999). Inducibility of atrial fibrillation during atrioventricular pacing with varying intervals: role of atrial 
electrophysiology and the autonomic nervous system. Journal of Cardiovascular Electrophysiology, 10(12), 1578-1585.

11. Roberts-Thomson, K. C., John, B., Worthley, S. G., Brooks, A. G., Stiles, M. K., Lau, D. H., Kuklik, P., Shipp, N. J., Kalman, J. M., \& Sanders, P. (2009). Left atrial remodeling in patients with atrial septal defects. Heart Rhythm, 6(7), 1000-1006.

12. Anne, W., Willems, R., Roskams, T., Sergeant, P., Herijgers, P., Holemans, P., et al. (2005). Matrix metalloproteinases and atrial remodeling in patients with mitral valve disease and atrial fibrillation. Cardiovascular Research, 67, 655-666.

13. Sanders, P., Morton, J. B., Davidson, N. C., Spence, S. J., Vohra, J. K., Sparks, P. B., et al. (2003). Electrical remodeling of the atria in congestive heart failure: electrophysiological and electroanatomic mapping in humans. Circulation, 108, 1461-1468.

14. Vaziri, S. M., Larson, M. G., Lauer, M. S., Benjamin, E. J., \& Levy, D. (1995). Influence of blood pressure on left atrial size. The Framingham Heart Study. Hypertension, 25, 1155-1160.

15. De Jong, A. M., Maass, A. H., Oberdorf-Maass, S. U., Van Veldhuisen, D. J., Van Gilst, W. H., \& Van Gelder, I. C. (2011). Mechanisms of atrial structural changes caused by stretch occurring before and during early atrial fibrillation. Cardiovascular Research, 89(4), 754-765.

16. Kuppahally, S. S., Akoum, N., Burgon, N. S., Badger, T. J., Kholmovski, E. G., Vijayakumar, S., et al. (2010). Left atrial strain and strain rate in patients with paroxysmal and persistent atrial fibrillation: relationship to left atrial structural remodeling detected by delayed-enhancement MRI. Circulation Cardiovascular Imaging, 3(3), 231-239.

17. Saha, S. K., Anderson, P. L., Caracciolo, G., Kiotsekoglou, A., Wilansky, S., Govind, S., et al. (2011). Global left atrial strain correlates with CHADS2 risk score in patients with atrial fibrillation. Journal of the American Society of Echocardiography, 24(5), 506512.

18. Shaikh, A. Y., Maan, A., Khan, U. A., Aurigemma, G. P., Hill, J. C., Kane, J. L., et al. (2012). Speckle echocardiographic left atrial strain and stiffness index as predictors of maintenance of sinus rhythm after cardioversion for atrial fibrillation: a prospective study. Cardiovascular Ultrasound, 10, 48.

19. Montserrat, S., Gabrielli, L., Bijnens, B., Borràs, R., Berruezo, A., Poyatos, S., et al. (2015). Left atrial deformation predicts success of first and second percutaneous atrial fibrillation ablation. Heart Rhythm, 12(1), 11-18.

20. Courtois, M., Fattal, P. G., Kovács, S. J., Jr., Tiefenbrunn, A. J., \& Ludbrook, P. A. (1995). Anatomically and physiologically based reference level for measurement of intracardiac pressures. Circulation, 92(7), 1994-2000.

21. Ishimatsu, T., Hayano, M., Hirata, T., Iliev, I. I., Komiya, N., Nakao, K., et al. (1999). Electrophysiological properties of the left atrium evaluated by coronary sinus pacing in patients with atrial fibrillation. Pacing and Clinical Electrophysiology, 22(12), 17391746.

22. Chen, M. C., Guo, G. B., \& Chang, H. W. (2002). Atrial electrophysiological properties evaluated by right and left atrial pacing in patients with or without atrial fibrillation. Japanese Heart Journal, 43(3), 231-240.

23. Lang, R. M., Bierig, M., Devereux, R. B., Flachskampf, F. A., Foster, E., Pellikka, P. A., Picard, M. H., Roman, M. J., Seward, J., Shanewise, J., Solomon, S., Spencer, K. T., St John Sutton, M., Stewart, W., \& American Society of Echocardiography's Nomenclature and Standards Committee; Task Force on Chamber Quantification; American College of Cardiology Echocardiography Committee; American Heart Association; European Association of Echocardiography; European Society of Cardiology. (2006).
Recommendations for chamber quantification. European Journal of Echocardiography, 7(2), 79-108.

24. Nagueh, S. F., Appleton, C. P., Gillebert, T. C., Marino, P. N., Oh, J. K., Smiseth, O. A., Waggoner, A. D., Flachskampf, F. A., Pellikka, P. A., \& Evangelista, A. (2009). Recommendations for the evaluation of left ventricular diastolic function by echocardiography. European Journal of Echocardiography, 10, 165-193.

25. Serri, K., Reant, P., Lafitte, M., Berhouet, M., Le Bouffos, V., Roudaut, R., \& Lafitte, S. (2006). Global and regional myocardial function quantification by two dimensional strain. Journal of the American College of Cardiology, 47, 1175-1181.

26. Mor-Avi, V., Lang, R. M., Badano, L. P., Belohlavek, M., Cardim, N. M., Derumeaux, G., Galderisi, M., Marwick, T., Nagueh, S. F., Sengupta, P. P., Sicari, R., Smiseth, O. A., Smulevitz, B., Takeuchi, M., Thomas, J. D., Vannan, M., Voigt, J. U., \& Zamorano, J. L. (2011). Current and evolving echocardiographic techniques for the quantitative evaluation of cardiac mechanics: ASE/EAE consensus statement on methodology and indications endorsed by the Japanese Society of Echocardiography. European Journal of Echocardiography, 12(3), 167-205.

27. Kurt, M., Wang, J., Torre-Amione, G., \& Nagueh, S. F. (2009). Left atrial function in diastolic heart failure. Circulation Cardiovascular Imaging, 2(1), 10-15.

28. Yoon, Y. E., Kim, H., Kim, S., Kim, S. H., Park, J., Park, K., Choi, S., Kim, M., Kim, H., \& Cho, G. (2012). Left atrial mechanical function and stiffness in patients with paroxysmal atrial fibrillation. Journal of Cardiovascular Ultrasound, 20(3), 140-145.

29. Chadaide, S., Domsik, P., Kalapos, A., Sághy, L., Forster, T., \& Nemes, A. (2013). Three-dimensional speckle tracking echocardiography-derived left atrial strain parameters are reduced in patients with atrial fibrillation (results from the MAGYAR-path study). Echocardiography, 30(9), 1078-1083.

30. Teh, A. W., Kistler, P. M., Lee, G., Medi, C., Heck, P. M., Spence, S., et al. (2011). Electroanatomic properties of the pulmonary veins: slowed conduction, low voltage and altered refractoriness in AF patients. Journal of Cardiovascular Electrophysiology, 22(10), 1083-1091.

31. Stiles, M. K., John, B., Wong, C. X., Kuklik, P., Brooks, A. G., Lau, D. H., et al. (2009). Paroxysmal lone atrial fibrillation is associated with an abnormal atrial substrate: characterizing the "second factor". Journal of the American College of Cardiology, 53(14), 1182-1191.

32. Teh, A. W., Kistler, P. M., Lee, G., Medi, C., Heck, P. M., Spence, S. J., et al. (2012). Electroanatomic remodeling of the left atrium in paroxysmal and persistent atrial fibrillation patients without structural heart disease. Journal of Cardiovascular Electrophysiology, 23(3), 232-238.

33. Lab, M. J. (1996). Mechanoelectric feedback (transduction) in heart: concepts and implications. Cardiovascular Research, 32(1), $3-14$.

34. Satoh, T., \& Zipes, D. P. (1996). Unequal atrial stretch in dogs increases dispersion of refractoriness conducive to developing atrial fibrillation. Journal of Cardiovascular Electrophysiology, 7, 833842.

35. Ravelli, F., Masè, M., del Greco, M., Marini, M., \& Disertori, M. (2011). Acute atrial dilatation slows conduction and increases AF vulnerability in the human atrium. Journal of Cardiovascular Electrophysiology, 22(4), 394-401.

36. Lau, C. P., Leung, W. H., Wong, C. K., \& Cheng, C. H. (1990). Haemodynamics of induced atrial fibrillation: a comparative assessment with sinus rhythm, atrial and ventricular pacing. European Heart Journal, 11(3), 219-224. 